\title{
A time domain decentralized algorithm for two channel active noise
}

\author{
control
}

\author{
Somanath Pradhan, ${ }^{\mathrm{a}}$ Guoqiang Zhang and Xiaojun Qiu
}

Centre for Audio, Acoustics and Vibration, Faculty of Engineering and IT, University of

Technology Sydney, Australia

\section{ABSTRACT}

Due to their low computational complexity, reduced wiring cost, and flexibility of scaling up, decentralized multiple channel active control systems are attractive in many applications. In a decentralized multiple channel active control system, a number of small subsystems are constructed, which are updated independently with only the associated error signals. In this letter, a time domain two channel decentralized control algorithm is proposed to achieve the similar noise reduction performance as the centralized one. Auxiliary filters are introduced to filter the reference signal for control filter update and a novel design method is proposed to shape the frequency response of the auxiliary filters. The simulation results using the measured impulse responses demonstrate the efficacy of the proposed algorithm for broadband noise control.

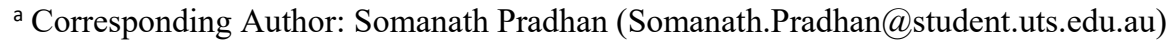




\section{INTRODUCTION}

Active noise control (ANC) technique has gained significant attention in mitigating noise by generating anti-noise using a control algorithm. The filtered-x least mean square (FxLMS) algorithm is the most commonly used algorithm in ANC applications due to its robustness and low computational complexity. ${ }^{1,2}$ To achieve global noise control, a centralized multiple channel ANC system can be employed, which requires many secondary path models for generating the filtered reference signals and all the error signals to update the control filters. When the number of channels increases, the computational complexity of the centralized algorithm increases significantly, and the complexity and cost of wiring and communication overhead between error sensors and the controller cause a big problem. ${ }^{3-5}$

Many approaches have been proposed to reduce computational complexity of multiple channel systems. Murao et al. proposed a mixed-error approach by combining all the error signals into one and used it for centralized control; however, the system possesses high communication load to feed all the error signals to the centralized controller. ${ }^{6}$ Alternatively, a distributed control approach has been proposed by considering each secondary source as a node in a ring network, in which the computational burden is distributed across all the nodes, but at the cost of high transmission bandwidth and delay. ${ }^{7}$

Due to their low computational complexity, reduced wiring cost, and flexibility of scaling up, decentralized multiple channel ANC systems are attractive in many applications, in which a number of smaller subsystems are employed to update the control filter independently with only the associated error signal. A study on a two channel frequency domain decentralized ANC (DANC) system shows that the system stability cannot be maintained if the control signals are not constrained in magnitude. ${ }^{8}$ A practical stability condition for decentralized feedback ANC systems 
has been derived by taking into account the geometrical configuration of secondary sources and error sensors. ${ }^{9}$ It has been found that reducing the number of channels and the distance between secondary loudspeakers and error microphones can increase system stability but at the cost of smaller noise reduction. ${ }^{10}$

Recently, it is shown that a two channel DANC system can achieve the same noise reduction performance as the centralized one by shaping the eigenvalues of a $2 \times 2$ matrix for each frequency bin properly such that they lie on the right complex domain. ${ }^{11}$ However, it only considers single frequency. An et al. proposed a time domain multiple channel DANC system for controlling periodic disturbances recently, but their method has two limitations. ${ }^{12}$ First, $N$ nonlinear equations are required to be solved to shape the eigenvalues of an $N \times N$ matrix for each frequency, which remains an open problem without knowing whether a solution exists or not; second, when converting the solution from frequency to time domain, the design of the auxiliary filter to filter the reference signal (to be used in the FxLMS algorithm) is complicated. The sensitive shaping parameters and the filter delay introduced in their system affect the convergence speed of the control algorithm.

In this letter, a novel two channel DANC framework in time domain is proposed for controlling broadband noise. Similarly to Ref. 12, the DANC solution in the frequency domain is obtained first and then the optimized time domain algorithm is developed. The novelties of this work are two-fold. First, the genetic algorithm (GA) is employed to compute the DANC solution in the frequency domain, where different frequency bins can have different convergence behaviors with the steepest descent algorithm. ${ }^{13}$ The solution obtained from the GA undergoes a scaling process so that different frequencies have roughly the similar convergence behaviors, which is crucial for broadband control. Second, a new and simple FIR filter design method is adopted for 
designing the auxiliary filters. The simulation results using the measured acoustic paths demonstrate the effectiveness of the proposed algorithm.

\section{THE PROPOSED ALGORITHM}

\section{A. Framework description}

Table I shows the framework of the proposed time domain two channel decentralized algorithm. The first step is to find a DANC solution in the frequency domain. To do so, a $2 \times 2$ frequency response matrix $\mathbf{S}$ of the secondary paths is constructed and then the GA is employed to obtain the diagonal matrix $\mathbf{C}$ of the DANC for each frequency. In principle, different step sizes can be used for controlling different frequency disturbances using the frequency domain steepest descent algorithm, whereas a single step size has to be used in full band time domain DANC algorithm. This poses a challenge in the system design. As described later, it is necessary to scale the $\mathbf{C}$ matrices to compensate for the different convergence behaviors across the frequencies. After that, the auxiliary FIR filters for filtering the reference signal are designed based on the obtained scaled C matrices.

TABLE I. Procedure of the proposed algorithm.

\footnotetext{
Step 1: $\quad$ Construct a $2 \times 2$ frequency response matrix $\mathbf{S}$ of the secondary paths for each frequency.

Step 2: $\quad$ Shape eigenvalue in frequency domain by finding a diagonal matrix $\mathbf{C}$ using the GA so that the eigenvalues of $\mathbf{C S}$ are at right complex domain.
} 


\begin{tabular}{ll}
\hline \hline Step 3: & Scale $\mathbf{C}$ for each frequency using Eq. (4) and (5) to balance the different \\
& convergence behaviors across the frequency bins. \\
Step 4: & Design the auxiliary FIR filter $R_{\mathrm{i}}(z)$ using Eq. (7). \\
Step 5: $\quad$ Carry out the control operation by updating the control filters using Eq. (8). \\
\hline
\end{tabular}

\section{B. Eigenvalue shaping in frequency domain}

Shaping eigenvalue with the GA is the second step. Following the same iterative learning rule for a two channel decentralized controller for each frequency, the input to the control sources can be computed iteratively as ${ }^{8}$

$$
\mathbf{y}(k+1)=\mathbf{y}(k)-[\mathbf{I}-\mu \mathbf{C S}] \mathbf{y}(k)-\mu \mathbf{C p},
$$

where $\mathbf{y}(k)$ is the input to control source at iteration $k$, p represents the primary disturbances at the error sensors, $\mathbf{I}$ is the identity matrix, $\mu$ is the step size, $\mathbf{C}=\operatorname{diag}\left(\left[c_{1}, c_{2}\right]\right)$, which is to be obtained, $\mathbf{S}$ denotes the $2 \times 2$ frequency response matrix of the secondary paths. To design a controller that achieves the optimal noise reduction performance, the stability condition is that the real part of the eigenvalues of the matrix $\mathbf{C S}$ must be positive. ${ }^{11}$ The diagonal matrix $\mathbf{C}$ can then be optimized to push the eigenvalues of $\mathbf{C S}$ to the right complex domain.

When $\mathbf{C}=\mathbf{S}^{\mathrm{H}}$, Eq. (1) represents the updating equation for the centralized controller. On the other hand, when $\mathbf{C}=\mathbf{S}_{\mathrm{d}}^{\mathrm{H}}\left(\mathbf{S}_{\mathrm{d}}^{\mathrm{H}}\right.$ is a diagonal matrix formed by taking the diagonal elements of $\left.\mathbf{S}\right)$, Eq. (1) represents the updating expression for the conventional DANC. In the following paragraph, the GA is used to shape the eigenvalues of $\mathbf{C S}$ appropriately to be at the right complex domain. ${ }^{14}$ To do so, the optimization for $\mathbf{C}$ can be formulated as 


$$
\mathbf{C}^{*}=\arg \min _{c} 1 \text { subject to } \mathbf{C}=\operatorname{diag}\left\{c_{1}, c_{2}\right\}
$$

$$
\text { and } \lambda_{i, \mathrm{Re}}(\mathbf{C S})>0, \quad i=1,2
$$

where 1 and $\lambda_{i, \mathrm{Re}}(\cdot)$ denote a constant function and real part of the $i$ th eigenvalue, respectively. Because it is difficult to apply the GA directly to solve Eq. (2), the above optimization problem has to be reformulated so that the objective function is differentiable. To start, $\mathbf{C}$ is assumed to be a product of two diagonal matrices and can be expressed as $\mathbf{C}=\operatorname{diag}\{\mathbf{a}\} \mathbf{S}_{\mathrm{d}}^{\mathrm{H}}$. Two functions $\theta_{\max }(a)$ $=\max _{i} \angle \lambda_{i}\left(\operatorname{diag}(\mathbf{a}) \mathbf{S}_{\mathrm{d}}^{\mathrm{H}}\right)$ and $\theta_{\min }(a)=\min _{i} \angle \lambda_{i}\left(\operatorname{diag}(\mathbf{a}) \mathbf{S}_{\mathrm{d}}^{\mathrm{H}}\right)$ are defined in the range $[-\pi \pi]$. Thus, the optimization problem can be reformulated as

$$
\mathbf{a}^{*}=\arg \min _{a}\left[\left(\theta_{\max }(\mathbf{a})\right)^{4} \times 1_{\theta_{\max }(\mathbf{a})>0}+\left(\theta_{\min }(\mathbf{a})\right)^{4} \times 1_{\theta_{\min }(\mathbf{a})<0}\right]
$$

subjected to $b_{l} \leq\left|a_{i}\right| \leq b_{u}$ and $0 \leq \angle a_{i} \leq 2 \pi$, where $1_{(\cdot)}$ is an indicator function, $b_{l}$ and $b_{u}$ are the positive lower and upper limits of the magnitude of elements of $\mathbf{a}$. The details for applying the GA to find the solution can be found in Ref. 13 .

\section{Scaling of $\mathbf{C}$ matrices}

For a DANC system in the frequency domain, the upper bound of step sizes for different frequencies are different when a steepest descent algorithm is employed, indicating that different frequencies exhibit different convergence behaviors. ${ }^{8,11}$ As the proposed algorithm is implemented in full band time domain, only one step size can be employed to incorporate the whole frequency of interest. To address the step size-inconsistency across the two domains, it is necessary to scale the obtained $\mathbf{C}$ matrices from Subsection II-B to mitigate the effect of the different convergence 
behaviors. In principle, $\mathbf{C}$ matrices can be scaled such that the resulting DANC system have roughly the same upper bound of step sizes across the frequencies.

Because it is time consuming to tune the scales of the $\mathbf{C}$ matrices manually, we propose to compute the scales mathematically in the following manner. Let the frequency response of the auxiliary filters $R_{\mathrm{i}}(\omega)(i=1,2)$ to be

$$
R_{i}(\omega)=\psi(\omega) \widehat{C}_{\mathrm{GA}, i}^{*}, \quad i=1,2
$$

where $\psi(\omega)>0$ denotes the positive scale for frequency $\omega$, and $\widehat{C}_{\mathrm{GA}, i}^{*}(\omega)$ is the $i$ th diagonal element of the solution for $\widehat{\mathbf{C}}_{\mathrm{GA}}$. The scale $\psi(\omega)$ is computed as

$$
\psi(\omega)=\left\|\widehat{\mathbf{C}}_{\mathrm{GA}}(\omega) \mathbf{S}(\omega)\right\|_{2} /\left\|\mathbf{S}^{\mathrm{H}}(\omega) \mathbf{S}(\omega)\right\|_{2} .
$$

It is found empirically that the expression in Eq. (5) can mitigate the effect of the different convergence behaviors.

\section{Auxiliary FIR filter design}

The frequency response of the auxiliary filter can be expressed in a compact form as $R_{i}(\omega)=$ $\mathbf{F}(\omega) \rho_{i}$, where $\mathbf{F}(\omega)=\left[1, \mathrm{e}^{-\mathrm{j} \omega}, \ldots, \mathrm{e}^{-\mathrm{j} \omega(L-1)}\right]$ is the transform vector and $\boldsymbol{\rho}_{i}=\left[\rho_{i} 0, \rho_{i 1}, \ldots, \rho_{i(L-1)}\right]^{\mathrm{T}}$ is the filter coefficient vector. Considering the real and imaginary parts for all the angular frequencies $\omega_{k}(k=1,2, \ldots, K)$, a linear equation can be constructed from Eq. (4) as 


$$
\left[\begin{array}{l}
\operatorname{Re}\left(\mathbf{F}\left(\omega_{1}\right)\right) \\
\vdots \\
\operatorname{Re}\left(\mathbf{F}\left(\omega_{K}\right)\right) \\
\operatorname{Im}\left(\mathbf{F}\left(\omega_{1}\right)\right) \\
\vdots \\
\operatorname{Im}\left(\mathbf{F}\left(\omega_{K}\right)\right)
\end{array}\right] \boldsymbol{\rho}_{i}=\left[\begin{array}{l}
\operatorname{Re}\left(\psi\left(\omega_{1}\right) \widehat{C}_{\mathrm{GA}, \mathrm{i}}^{*}\left(\omega_{1}\right)\right) \\
\vdots \\
\operatorname{Re}\left(\psi\left(\omega_{K}\right) \widehat{C}_{\mathrm{GA}, \mathrm{i}}^{*}\left(\omega_{K}\right)\right) \\
\operatorname{Im}\left(\psi\left(\omega_{1}\right) \widehat{C}_{\mathrm{GA}, \mathrm{i}}^{*}\left(\omega_{1}\right)\right) \\
\vdots \\
\operatorname{Im}\left(\psi\left(\omega_{K}\right) \hat{C}_{\mathrm{GA}, \mathrm{i}}^{*}\left(\omega_{K}\right)\right)
\end{array}\right],
$$

where $\operatorname{Re}(\cdot)$ and $\operatorname{Im}(\cdot)$ denotes the real and imaginary parts, respectively. Denoting the first matrix of the left hand side of Eq. (6) as $\mathbf{A}$ and the right hand side of Eq. (6) as vector $\mathbf{b}$, it can be expressed as $\mathbf{A} \boldsymbol{\rho}_{i}=\mathbf{b}$. The optimum solution for $\boldsymbol{\rho}_{i}$, which is the filter coefficient vector of the $i$ th auxiliary filter, can be obtained as

$$
\boldsymbol{\rho}_{\mathbf{i}}=\left(\mathbf{A}^{\mathrm{H}} \mathbf{A}\right)^{-1} \mathbf{A}^{\mathrm{H}} \mathbf{b} \text {. }
$$

Unlike the auxiliary filter design method reported in Ref. 12, this proposed method does not include any additional delay in the filter, i.e., the effect of the additional delay on convergence speed of the control algorithm is mitigated. Here, the length of the auxiliary filter $L$ is the same as the length of secondary paths $L_{\mathrm{s}}$.

\section{E. The time domain control algorithm}

Figure 1 depicts the schematic diagram of the proposed algorithm, where $x(n)$ is the reference signal, $y_{i}(n)$ is the $i$ th $(i=1,2)$ control signal and $W_{i}(z)$ denotes the transfer function of the $i$ th control filter, $p_{j}(n)$ is the primary disturbance at the $j$ th $(j=1,2)$ error sensor, $S_{i j}(z)$ denotes the acoustic transfer function from the $i$ th secondary source to the $j$ th error sensor and $s_{i j}(n)$ denotes its corresponding impulse response and $e_{j}(n)$ is the residual error signal at the $j$ th error sensor. 

signal using the FxLMS algorithm as

$$
\mathbf{w}_{i}(n+1)=\mathbf{w}_{i}(n)-\mu \mathbf{r}_{i}(n) e_{i}(n),
$$

Fig. 1. Schematic diagram of the proposed time domain two channel decentralized algorithm.

where $\mathbf{w}_{i}(n)$ is the $i$ th control filter coefficient vector, $\mu$ is the step size parameter, and $\mathbf{r}_{i}(n)=\left[r_{i}(n)\right.$, $\left.r_{i}(n-1), \ldots, r_{i}\left(n-L_{w}+1\right)\right]^{\mathrm{T}}$ is the tap delayed vector of the filtered reference signal $r_{i}(n)$ for the $i$ th control filter with $L_{\mathrm{w}}$ denoting the length of control filter.

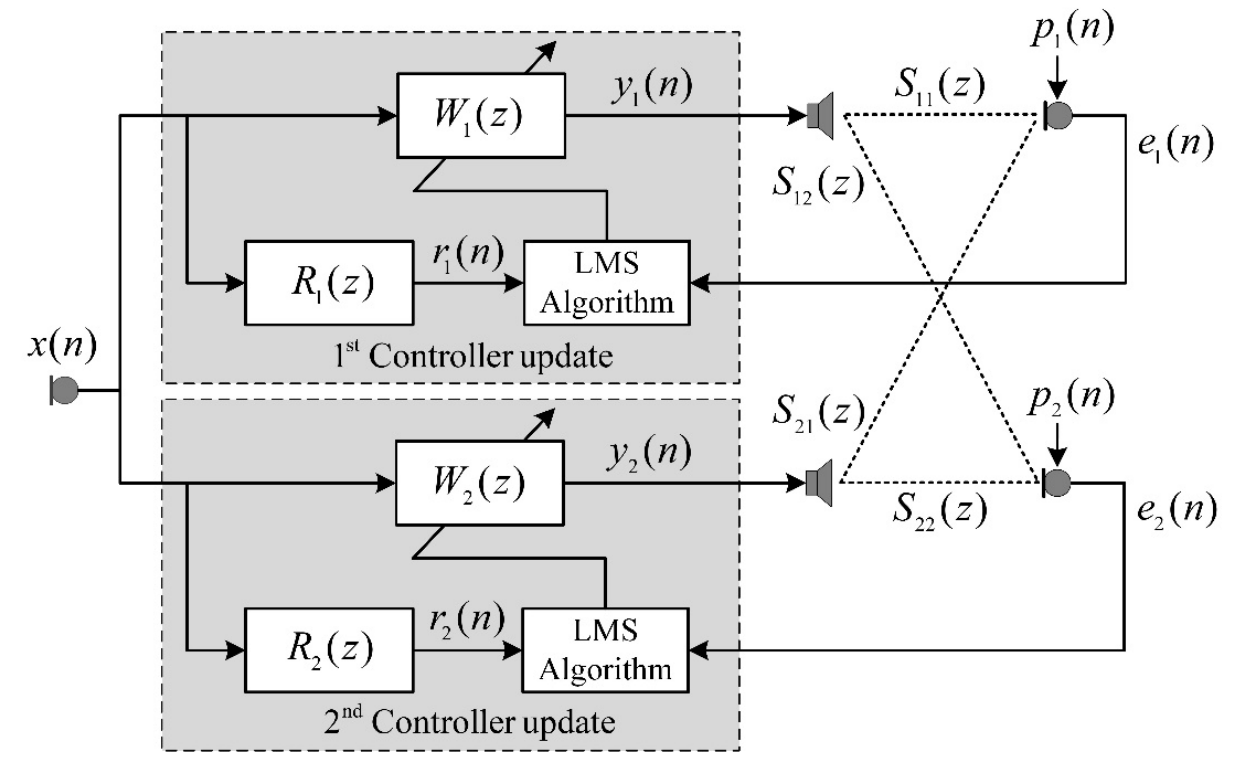




\section{SIMULATIONS}

In this section, simulations are carried out to demonstrate the noise reduction performance of the proposed algorithm as compared to the conventional time domain decentralized FxLMS algorithm, the centralized FxLMS algorithm, and the mixed-error approach reported in Ref. 6. In the simulations, the primary paths and secondary paths are FIR filters of length 256 and 128, respectively, which were measured in a normal room at the Tech Lab of University of Technology Sydney. The space between the centers of the two secondary loudspeakers was $0.1 \mathrm{~m}$; the primary noise source was placed at $1.0 \mathrm{~m}$ away from the secondary sources; the distances from the center of the secondary loudspeakers to their respective error microphones was set as $0.1 \mathrm{~m}$. The primary and secondary paths were obtained with a white noise excitation. Each of the control filter $W_{i}(z)$ is considered as 256-tap FIR. The sampling frequency used in the simulation is $4 \mathrm{kHz}$. All the simulation results are ensembled over 50 independent trials and smoothed by moving average method using a window of 256 samples. The normalized mean square error (MSE) is used as the metric for comparison. ${ }^{12}$

First, the values of $\widehat{C}_{\mathrm{GA}, i}^{*}(\omega)$ are obtained from the GA for frequencies ranging from $1 \mathrm{~Hz}$ to $2000 \mathrm{~Hz}$ with an incremental step of $1 \mathrm{~Hz}$ and the corresponding scale parameters $\psi(\omega)$ are calculated. The filter for the $i$ th auxiliary filter is obtained as a 128-tap FIR filter $(L=128)$. The $i$ th control filter is updated using the $i$ th filtered reference signal and $i$ th error signal following the learning rule in Eq. (8). Two types of noises are considered for the simulation, where the first one is a white noise and the second is a traffic noise recorded from a highway. A white Gaussian measurement noise with signal to noise ratio (SNR) of $40 \mathrm{~dB}$ is considered to mimic a practical environment. 
Figure 2 depicts the normalized MSE curves for a zero-mean white Gaussian noise with unit

189

190

191

192

193 variance, where the primary path changes after $100 \mathrm{~s}$. The variation in the primary path was obtained by shifting the primary noise source by $0.2 \mathrm{~m}$ towards the control sources and then rotated clockwise by an angle of $30^{\circ}$ and pointed towards the secondary sources for demonstrating the tracking performance of the control filters. One can observe from Fig. 2(a) that the conventional decentralized algorithm with the maximum possible step size $\mu=4 \times 10^{-7}$ (without stability issue) achieves a noise reduction of around $11 \mathrm{~dB}$ with a slow convergence. A higher value of step size results in algorithmic divergence for the conventional decentralized algorithm, which can be observed from Fig. 2(b).

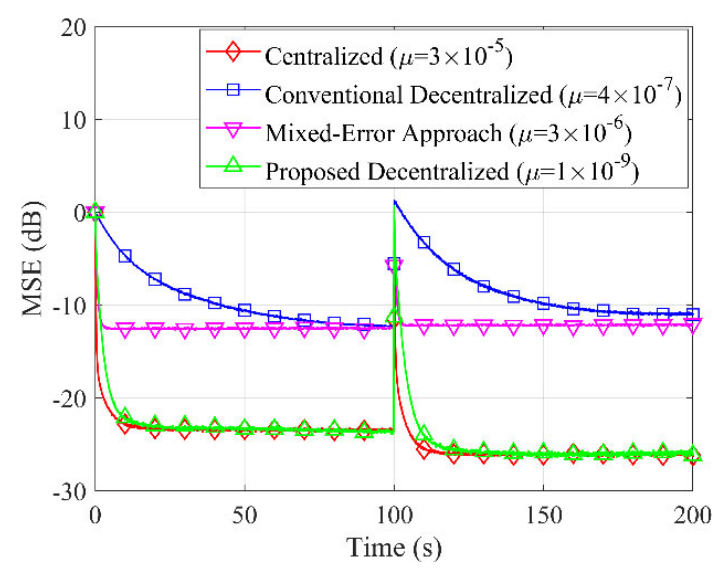

(a)

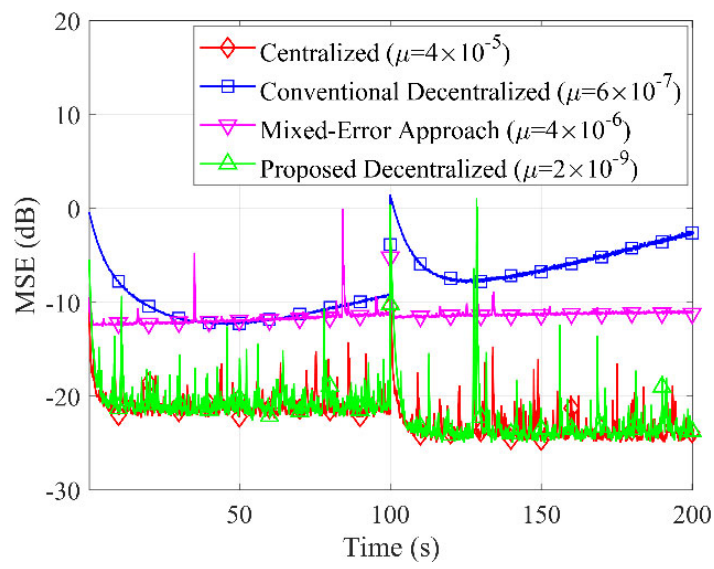

(b)

Fig. 2. Normalized MSE curves for zero-mean white Gaussian noise using different algorithms when they (a) converge and (b) diverge.

The proposed decentralized algorithm with its maximum possible step size $\mu=1 \times 10^{-9}$ achieves around 23-26 $\mathrm{dB}$ noise reduction with a faster convergence speed, whereas the mixed-error approach with step size $\mu=3 \times 10^{-6}$ achieves around $12 \mathrm{~dB}$ noise reduction, whose control 
performance is better than the conventional decentralized algorithm but not as good as the proposed algorithm. The centralized algorithm with maximum step size $\mu=3 \times 10^{-5}$ achieves around 23-26 dB noise reduction with the fastest convergence among the four algorithms. The step sizes of the proposed algorithm and centralized one in Fig. 2(a) are chosen by trial and error in such a way that they achieve similar steady state noise reduction without any stability issue. The step sizes of the conventional decentralized algorithm and the mixed-error approach in Fig. 2(a) are also chosen by trial and error to provide the best possible noise reduction. Higher values of step sizes for the four algorithms compared to the chosen values cause algorithmic divergence or stability issue as shown in Fig. 2(b). It is clear that the upper bound step size for the centralized algorithm is larger than that for the other algorithms.

Figure 3 shows the results for the traffic noise recorded from a highway. The normalized MSE curves for this case are depicted in Fig. 3(a), and the power spectral density (PSD) of the sum of two residual error signals with and without control are shown in Fig. 3(b). The conventional decentralized algorithm performs the worst, and the noise reduction performance of the conventional decentralized algorithm deteriorates significantly from $500 \mathrm{~Hz}$ to $1500 \mathrm{~Hz}$ and there is little control above $1500 \mathrm{~Hz}$. The mixed-error approach is better. The proposed decentralized algorithm and the centralized algorithm perform the best with similar noise reduction. The step sizes of the four algorithms are chosen in the similar way as that for the white noise case. The strength of the proposed algorithm is that each controller only uses its own (nearest) error signal for update, this avoids processing and wiring for other error signals. 


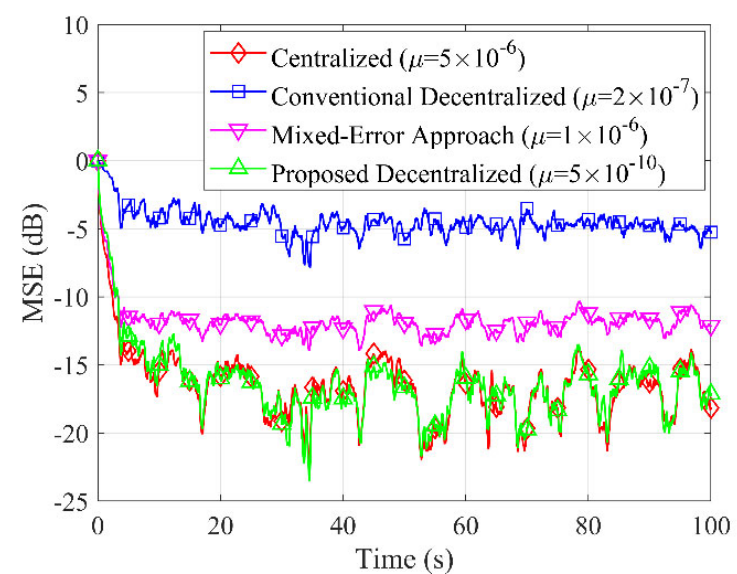

(b)

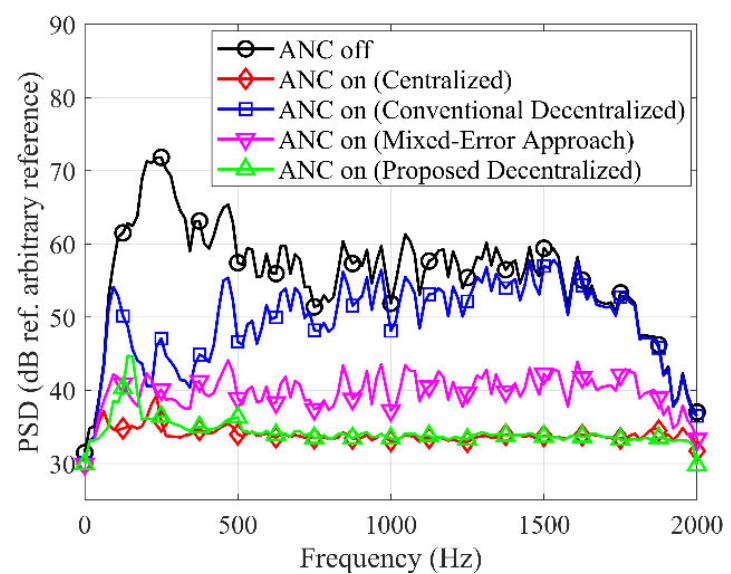

(b)

Fig. 3. (a) Normalized MSE curves for traffic noise using different algorithms and (b) the power spectral density with and without noise control.

The proposed algorithm for the two channel DANC requires $4 L_{\mathrm{w}}+2 L_{\mathrm{s}}+2$ multiplications per sample and $4 L_{\mathrm{w}}+2 L_{\mathrm{s}}-4$ additions per sample. Table II presents the computation complexity of the 4 algorithms, and an example is provided for straight forward comparison, where $L_{\mathrm{w}}=256$ and $L_{\mathrm{s}}=L=128$. It can be observed that the computational complexity of the proposed algorithm is same as the conventional decentralized algorithm and the mixed error approach, and it is less than its centralized counterpart. In addition to high computational complexity, the centralized ANC system has the highest cost of wiring and the largest communication overhead compared to other algorithms. Despite having vested with reduced complexity, the mixed error approach still needs to communicate with the two error sensors for each control filter update. It is worth noting that the mixed error approach uses mixed secondary path estimates, which are the transfer functions from the $i$ th secondary source to the mixed error signal. ${ }^{6}$ The conventional DANC system and the DANC system with the proposed algorithm require the least cost of wiring and communication overhead; nevertheless, the proposed algorithm requires some preprocessing of the estimated secondary paths 
before control operation. It is worth noting that the secondary paths are assumed to be perfectly estimated offline in advance before being used in the algorithm. If the secondary paths change drastically, re-estimation of secondary paths is required followed by the preprocessing to design the auxiliary filters. The variation of secondary paths might affect the performance of the system, which will be investigated in the future.

TABLE II. Computational complexity per sample of different algorithms.

\begin{tabular}{lcccc}
\hline \hline Algorithms & Multiplication (×) & Addition $(+)$ & \multicolumn{2}{c}{ Example } \\
\cline { 3 - 5 } & & & $(\times)$ & $(+)$ \\
\hline Centralized & $6 L_{\mathrm{w}}+4 L_{\mathrm{s}}+4$ & $6 L_{\mathrm{w}}+4 L_{\mathrm{s}}-6$ & 2052 & 2042 \\
Conventional decentralized & $4 L_{\mathrm{w}}+2 L_{\mathrm{s}}+2$ & $4 L_{\mathrm{w}}+2 L_{\mathrm{s}}-4$ & 1282 & 1276 \\
Mixed-error Approach & $4 L_{\mathrm{w}}+2 L_{\mathrm{s}}+2$ & $4 L_{\mathrm{w}}+2 L_{\mathrm{s}}-4$ & 1282 & 1276 \\
Proposed & $4 L_{\mathrm{w}}+2 L_{\mathrm{s}}+2$ & $4 L_{\mathrm{w}}+2 L_{\mathrm{s}}-4$ & 1282 & 1276 \\
\hline
\end{tabular}

251

252

\section{CONCLUSION}

In this work, a time domain decentralized adaptive control algorithm is proposed for the two channel ANC system. The frequency responses of the auxiliary filters are optimized using the GA followed by a scaling process. Unlike the existing methods, a simplified filter design method is developed. The simulation results with the measured acoustic paths demonstrate that the proposed algorithm is able to achieve similar noise reduction performance as the centralized algorithm. The convergence behavior and noise reduction performance of the proposed algorithm is better than the conventional decentralized algorithm and the mixed-error approach despite having the fact that 
the upper bound step size for the proposed algorithm is smaller than that for the centralized algorithm. Future work includes extending the proposed algorithm to multichannel ANC systems with large channel number $(>2)$ for broadband noise control.

\section{ACKNOWLEDGEMENT}

This work was supported by the Australian Research Council's Linkage Project, grant number LP160100616.

\section{REFERENCES}

${ }^{1}$ S. M. Kuo and D. R. Morgan, Active noise control systems (Wiley, New York, 1996).

${ }^{2}$ S. J. Elliott, Signal processing for active control (Academic Press, London, 2001).

${ }^{3}$ S. M. Kuo and D. R. Morgan, "Active noise control: a tutorial review," Proceedings of the IEEE 87 (6), 943-973 (1999).

${ }^{4}$ N. V. George and G. Panda, "A particle-swarm-optimization-based decentralized nonlinear active noise control system,” IEEE Trans. Instrum. Meas. 61 (12), 3378-3386 (2012).

${ }^{5}$ D. Shi, W.-S. Gan, J. He and B. Lam, "Practical implementation of multichannel filtered-x least mean square algorithm based on the multiple-parallel-branch with folding architecture for largescale active noise control,” IEEE Trans. Very Large Scale Integ. (VLSI) Systems (published online 2019).

${ }^{6}$ T. Murao, C. Shi, W.-S. Gan and M. Nishimura, "Mixed-error approach for multi-channel active noise control of open windows,” Appl. Acoust. 127, 305-315 (2017).

${ }^{7}$ M. Ferrer, M. de Diego, G. Piñero and A. Gonzalez, "Active noise control over adaptive 
distributed networks," Signal Process. 107, 82-95 (2015).

${ }^{8}$ S. J. Elliott and C. C. Boucher, "Interaction between multiple feedforward active control systems," IEEE Trans. Speech Audio Process. 2 (4), 521-530 (1994).

${ }^{9}$ E. Leboucher, P. Micheau, A. Berry and A. L’Espérance, “A stability analysis of a decentralized adaptive feedback active control system of sinusoidal sound in free space," J. Acoust. Soc. Am. 111 (1), 189-199 (2002).

${ }^{10}$ J. Tao, S. Wang, X. Qiu and J. Pan, "Performance of an independent planar virtual sound barrier at the opening of a rectangular enclosure," Appl. Acoust. 105, 215-223 (2016).

${ }^{11}$ G. Zhang, J. Tao, X. Qiu and I. Burnett, "Decentralized two-channel active noise control for single frequency by shaping matrix eigenvalues," IEEE/ACM Trans. Audio Speech Lang. Process. 27 (1), 44-52 (2018).

${ }^{12}$ F. An, Y. Cao and B. Liu, "Optimized decentralized adaptive control of noise and vibration for periodic disturbances," J. Acoust. Soc. Am. 144 (4), EL275-EL280 (2018).

${ }^{13}$ G. Zhang, J. Tao and X. Qiu, "Empirical Study of Decentralized Multi-Channel Active Noise Control Based on the Genetic Algorithm," in proceedings of the 23rd International Congress on Acoustics, Aachen, Germany (2019), pp. 6913-6920.

${ }^{14}$ D. E. Goldberg, Genetic algorithms. (Pearson Education India, 2006). 